\title{
Matching-Adjusted Indirect Comparison of Efficacy and Consumption of rVIII-SingleChain Versus Two Recombinant FVIII Products Used for Prophylactic Treatment of Adults/Adolescents with Severe Haemophilia A
}

Santiago Bonanad · Ramiro Núñez · Jose Luis Poveda $\cdot$ Karin Kurnik · Georg Goldmann •

Valeska Andreozzi $\cdot$ Björn Vandewalle $\cdot$ Sandra Santos

Received: May 21, 2021 / Accepted: June 30, 2021 / Published online: August 8, 2021

(C) The Author(s) 2021

\section{ABSTRACT}

Introduction: Given the relatively small number of patients with haemophilia A, head-tohead comparisons between recombinant FVIII (rFVIII) products are difficult to conduct. This study compared the efficacy and consumption of rVIII-SingleChain (lonoctocog alfa, AFSTYLA $^{\circledR}$ ) with rAHF-PFM (octocog alfa, Advate $^{\circledR}$ ) and rFVIIIFc (efmoroctocog alfa, Elocta $\left.^{\circledR}\right)$, for the prophylaxis and treatment of

Supplementary Information The online version contains supplementary material available at https:// doi.org/10.1007/s12325-021-01853-0.

S. Bonanad · J. L. Poveda

Hospital Universitari i Politècnic La Fe, Valencia, Spain

\section{R. Núñez}

Hospital Virgen del Rocío, Seville, Spain

K. Kurnik

Dr. Von Haunersches Kinderspital Klinikum der

Universität München, Munich, Germany

G. Goldmann

Institute of Experimental Haematology and

Transfusion Medicine, University of Bonn, Bonn, Germany

V. Andreozzi · B. Vandewalle $(\bowtie)$

Exigo Consultores, Lisbon, Portugal

e-mail: bjorn.vandewalle@exigoconsultores.com

S. Santos

CSL Behring, Lisbon, Portugal bleeding episodes in previously treated adolescents/adults with severe haemophilia A, through a matching-adjusted indirect comparison (MAIC).

Methods: A systematic literature review identified published clinical trials for rAHF-PFM and rFVIIIFc. Individual patient data for rVIII-SingleChain were used to match baseline patient characteristics to those from published trials, using an approach similar to propensity score weighting. After matching, annualized bleeding rates (ABR), percentage of patients with zero bleeds, and rFVIII consumption were compared across trial populations.

Results: Published data were identified from two rAHF-PFM trials and one rFVIIIFc trial. rVIII-SingleChain had similar ABR (risk ratio [RR]: 0.74 [0.16; 3.48]; RR: 1.18 [0.85; 1.65]) and percentage of patients with zero bleeds (odds ratio [OR]: 1.34 [0.56; 3.22]; OR: 0.78 [0.47; 1.31]) versus rAHF-PFM and rFVIIIFc, respectively. Annual rVIII-SingleChain consumption was significantly lower than rAHF-PFM (mean difference: - 1507.66 IU/kg/year [- 2011.71; $-1003.61]$ ) and equivalent to rFVIIIFc (RR: 0.96 $[0.62 ; 1.49])$.

Conclusion: Although limited to published information for comparator trials, these results suggest that with an annualized rFVIII consumption comparable to rFVIIIFc, but significantly lower than rAHF-PFM, routine prophylaxis with rVIII-SingleChain is able to maintain a similar $\mathrm{ABR}$ and percentage of 
patients with zero bleeds, attesting to the longacting nature of rVIII-SingleChain.

\section{PLAIN LANGUAGE SUMMARY}

It is difficult to directly compare different recombinant FVIII products in head-to-head studies because there are few patients with haemophilia A. This study aimed to indirectly compare the efficacy and consumption of different recombinant FVIII products in the prophylactic treatment of haemophilia A using published clinical data. A proven method for performing indirect comparisons of products is referred to as a matching-adjusted indirect comparison. Using this approach, we were able to compare rVIII-SingleChain with two other recombinant FVIII products (rAHF-PFM and rFVIIIFc). Our results suggest that annual FVIII consumption with rVIII-SingleChain is comparable to rFVIIIFc, but is significantly lower than rAHF-PFM, while maintaining a similar bleeding rate. These results highlight the long-acting nature of the product.

Keywords: Annualized bleeding rate; Efmoroctocog alfa; FVIII consumption; Haemophilia A; Lonoctocog alfa; Matchingadjusted indirect comparison; MAIC; Octocog alfa; rVIII-SingleChain

\section{Key Summary Points}

Why carry out the study?

Prophylaxis with recombinant factor VIII (rFVIII) replacement therapy is the recommended and most widely accepted treatment strategy to reduce bleeding risks in patients with severe haemophilia A.

Standard-acting rFVIII products like rAHFPFM have a short half-life leading to the need for injection intervals of 2-3 days to maintain sufficiently high factor activity levels to reduce the risk of bleeding.
Long-acting rFVIII products, such as rVIIISingleChain and rFVIIIFc, have been designed with improved pharmacokinetic properties to enable longer intervals between injections; these long-acting rFVIII products allow patients to maintain, or even decrease, their bleeding rates, whilst reducing the injection burden.

This study used matching-adjusted indirect comparison (MAIC) to compare the efficacy and consumption of rVIIISingleChain versus rAHF-PFM and rFVIIIFc.

\section{What was learned from the study?}

Prophylactic treatment with rVIIISingleChain has comparable efficacy to rAHF-PFM but significantly lower consumption. Comparable efficacy and consumption were established when rVIIISingleChain was compared to rFVIIIFc.

This indirect treatment comparison suggests that with an annualized rFVIII consumption comparable to rFVIIIFc, but significantly lower than rAHF-PFM, routine prophylaxis with rVIII-SingleChain is able to maintain a similar annualized bleeding rate and percentage of patients with zero bleeds, attesting to the long-acting nature of rVIII-SingleChain.

\section{INTRODUCTION}

Prophylaxis with plasma-derived or recombinant factor VIII (FVIII) replacement therapy is the recommended and most widely accepted treatment strategy to reduce the risk of bleeding and chronic arthropathy in adult patients with severe haemophilia A [1-3]. Although the World Federation of Hemophilia does not express a preference for recombinant over plasma-derived replacement therapies, over the last three decades, in many European countries, recombinant FVIII (rFVIII) replacement 
therapies have become the main FVIII products used for treatment [3-5].

A broadly used and established rFVIII product, with over 15 years of real-world treatment experience and proven clinical performance, is the plasma and albumin-free anti-haemophilic factor rAHF-PFM (octocog alfa, Advate ${ }^{\circledR}$, Shire). It was the first third-generation recombinant rFVIII product to receive marketing authorization by the European Medicines Agency (EMA) for the prophylaxis of bleeding episodes in haemophilia A [6, 7]. In adult patients with haemophilia $\mathrm{A}$, despite intra- and interindividual variations, on average, standard-acting rFVIII products like rAHF-PFM have a plasma half-life of approximately $12 \mathrm{~h}$ [8]. This leads to the need for intravenous rFVIII injections at intervals of 2-3 days to maintain factor activity levels sufficiently high to reduce the risk of bleeding. Intense treatment regimens like these pose a non-negligible burden to all patients, especially those with poor venous access, potentially reducing long-term therapeutic compliance and adherence. Lack of compliance and adherence to FVIII prophylaxis have long been recognized as key determinants of increased bleeding $[9,10]$.

With the aim of reducing the burden of frequent intravenous rFVIII injections for prophylactic treatment regimens, several rFVIII products have been designed with improved pharmacokinetic (PK) properties to allow for longer intervals between injections. These longacting rFVIII products allow patients to maintain, or even decrease, their bleeding rates, whilst reducing the injection burden [11].

The first long-acting third-generation rFVIII product to receive marketing authorization by the EMA for the prophylaxis of bleeding episodes in haemophilia A, and enter the European market in 2015, was the recombinant human FVIII Fc fusion protein, rFVIIIFc (efmoroctocog alfa, Elocta ${ }^{\circledR}$, Sobi) $[12,13]$. In adult patients with severe haemophilia $A$, the half-life of rFVIIIFc was estimated to be about $50 \%$ longer than that of standard-acting rFVIII products, allowing the intravenous rFVIII injection frequency to be extended to every 3-5 days [14].
A more recent long-acting third-generation rFVIII product licenced in Europe by the EMA for the prophylaxis of bleeding episodes in haemophilia A is rVIII-SingleChain (lonoctocog alfa, AFSTYLA ${ }^{\circledR}$, CSL Behring), developed using single-chain technology to improve the PK profile of rFVIII [11]. rVIII-SingleChain was extensively studied in the AFFINITY clinical program, comprising phase I/III studies investigating the PK, safety and efficacy in the treatment of bleeding episodes, routine prophylaxis, and surgical prophylaxis of previously treated patients with severe haemophilia A [15-18]. rVIII-SingleChain has demonstrated a modest increase in half-life as compared to standard-acting rFVIII products [15]. It has been shown to have a strong affinity to von Willebrand factor (VWF) [19], resulting in a lower clearance rate than standard-acting rFVIII and leading to a substantial increase in the area under the curve (AUC) [15]. The resulting greater drug exposure allows for longer dosing intervals than indicated by the half-life of rVIII-SingleChain alone, suggesting that it qualifies as a long-acting product; these features demonstrate that care should be taken when comparing rFVIII products by single PK parameters [11, 20]. Even when multiple PK parameters are considered simultaneously for treatment decisions, they are still surrogate markers for the actual clinical outcomes of interest. Besides budgetary considerations related to rFVIII product consumption, the key clinical consideration should be the treatment effect on bleeding rates, ideally targeting a rate of zero bleeds $[3,21]$.

There is a clear need to better understand the efficacy and factor consumption of rVIIISingleChain as routine prophylaxis in previously treated adolescents/adults with severe haemophilia A, as compared to other established and/or emerging rFVIII products. In the absence of head-to-head randomized controlled trials (RCT), an indirect treatment comparison of the annualized bleeding rate $(\mathrm{ABR})$, percentage of patients with zero bleeds and annualized rFVIII consumption of rVIIISingleChain versus rAHF-PFM and rFVIIIFc was performed. 


\section{METHODS}

\section{Data Sources and Sample Selection}

Individual patient-level data (IPD) from previously treated adolescents/adults enrolled in the pivotal, prospective, open-label, non-randomized, multicentre phase I/III clinical trial of rVIII-SingleChain as part of the AFFINITY clinical program [16] were provided by the trial sponsor.

To identify published clinical trials of rAHFPFM and rFVIIIFc for the prophylaxis of bleeding episodes among previously treated patients with severe haemophilia $\mathrm{A}$, a systematic literature review was conducted using three electronic databases (Medline, Cochrane CENTRAL and Web of Science). Screening was performed by three independent researchers using five selection levels: duplicate removal, title reading, abstract reading, full-text reading (qualitative analysis) and eligibility for matching-adjusted indirect comparison (MAIC) (quantitative analysis). The screening criteria for the systematic literature review are detailed in Supplementary Material Table S1.

The treatment arms from the different studies that presented a prophylaxis regimen aligned with the regimen of routine prophylaxis recommended in the European Summary of Product Characteristics of rVIII-SingleChain (20-50 IU/kg, two to three times weekly), rAHFPFM (20-40 IU $/ \mathrm{kg}$, at intervals of 2-3 days) and rFVIIIFc (25-65 IU/kg, every 3-5 days) were selected for comparison [8, 12, 22].

For the included studies, information on study design and data on all available baseline characteristics and outcomes of interest were extracted from the selected treatment arms. Outcomes of interest were the ABR, percentage of patients with zero bleeds, and annualized rFVIII consumption (IU/kg/year).

The phase I/III clinical trial of rVIII-SingleChain was conducted in accordance with the International Conference on Harmonization Guidelines for Good Clinical Practice and the ethical principles outlined in the Declaration of Helsinki 2008. Ethics approval, individual informed consent, and approval by the relevant national authorities were obtained prior to enrolment. Informed consent was not required for this analysis given the deidentified nature of the AFFINITY individualized patient-level data and the use of anonymized, previously published data for rAHF-PFM and rFVIIIFc studies.

\section{Statistical Methods}

To perform the indirect comparisons of rVIIISingleChain versus rAHF-PFM and rFVIIIFc, in the absence of head-to-head RCT and common control arms, a MAIC approach was used. MAIC is a validated population-adjustment method that ensures comparisons are conducted between more balanced patient populations than would be the case with naïve indirect comparisons [23, 24].

Using IPD, the influence of patients from the rVIII-SingleChain prophylaxis arm of the phase I/III trial was reweighted, such that the weighted average of their baseline characteristics matched the aggregate baseline characteristics of the selected prophylaxis arms of the comparator trials. Individual weights were calculated by a propensity score-type logistic regression model, contemplating matching variables selected on the basis of their availability in both the rVIII-SingleChain phase I/III trial and comparator trials.

Using the same weights to balance the trial populations, weighted outcomes (and corresponding confidence intervals) were estimated from the IPD of the rVIII-SingleChain phase I/ III trial. This eliminates potential confounding due to observed trial population differences, hence allowing for direct statistical comparison between the reweighted outcomes and precision of the rVIII-SingleChain phase I/III trial and the observed outcomes and precision from the comparator trials.

Calculation of endpoints by different techniques in the different comparator trials was accommodated for, by applying the same techniques to the IPD of the rVIII-SingleChain phase I/III trial $[14,25,26]$. Missing precision measures for outcomes in the comparator trials were assumed to be equal to the ones estimated for the corresponding outcomes in the rVIII- 
SingleChain phase I/III trial. No pooling of information was performed.

All statistical analyses were performed using the $\mathrm{R}$ statistical computing software [27], published code from the National Institute of Health and Care Excellence (NICE) [24] and a $5 \%$ significance level.

\section{RESULTS}

\section{Data Sources and Sample Selection}

For rVIII-SingleChain, the IPD from the routine prophylaxis arm of the adolescent/adult patient population of the phase I/III trial (NCT01486927, Parts 2 and 3) [16] were selected. Routine prophylaxis was prescribed as 20-40 IU/kg rVIII-SingleChain every second day or $20-50 \mathrm{IU} / \mathrm{kg}$ rVIII-SingleChain two to three times per week, or at other doses/frequencies at the investigator's discretion [16].

Three published clinical trials were identified from the systematic literature search for indirect comparison through MAIC: two rAHF-PFM trials (Tarantino et al. 2004 [28] and Valentino et al. 2012 [25]) and one rFVIIIFc trial (A-LONG, Mahlangu et al. 2014 [14]). The PRISMA flow diagram for the systematic literature review is detailed in Supplementary Material Fig. S1.

For the rAHF-PFM clinical trial published in 2004 (rAHF-PFM-2004), aggregate data was considered from the patients subjected to at least 75 exposure days of protocol-specified prophylaxis (Part 2), consisting of 25-40 IU/kg of rAHF-PFM administered three times per week or every other day, or other doses at the discretion of the investigator [28].

From the 2012 published rAHF-PFM clinical trial (rAHF-PFM-2012), aggregate data from the standard prophylaxis arm was used, based on common clinical practice, with every other day administrations of rAHF-PFM at doses of 20-40 IU/kg [25].

Aggregate data for rFVIIIFc was obtained from the individualized prophylaxis arm of the 2014 published A-LONG study, consisting of 25-65 IU/kg administered every 3-5 days, targeting FVIII levels as to maintain good control of breakthrough bleeds [14].

\section{Patient Populations}

A total of 146 patients from the rVIII-SingleChain routine prophylaxis arm of the phase I/III trial were considered in the analysis, with a mean efficacy evaluation period of 244 days. For the comparison of annualized rFVIII consumption, one patient was excluded from the analysis because of missing data on consumption.

The rAHF-PFM-2004 trial consisted of 111 patients, 108 of which received study drug (mean total rAHF-PFM exposure of 117 days) and 107 were treated for at least 75 exposure days with protocol-specified prophylaxis. Baseline characteristics in common between the rVIII-SingleChain and rAHF-PFM-2004 populations are summarized in Table 1 . In general, the rVIII-SingleChain population included a higher proportion of adult patients and a lower proportion of white patients (Table 1) [16, 28].

The standard prophylaxis arm of the rAHFPFM-2012 trial included 32 patients, with a mean treatment period of 362 days. As compared to the rAHF-PFM-2012 population, the rVIII-SingleChain population included a lower proportion of white patients and a slightly higher proportion of patients aged 16 years or above (Table 2) [16, 25].

Baseline characteristics in common between the rVIII-SingleChain population and the rFVIIIFc individualized prophylaxis population of the A-LONG trial, including 118 patients with a median duration of treatment of 225 days, were aligned, with the exception of a higher percentage of European patients in the rVIII-SingleChain population (Table 3) $[14,16]$.

Tables 1, 2 and 3 also show that after reweighting the influence of patients from the rVIII-SingleChain population, the summary statistics of all matched baseline characteristics were exactly balanced for each of the comparator populations.

\section{Indirect Comparisons}

Different endpoints were calculated by different techniques in the comparator trials (e.g. square root transformation of ABR in the rAHF-PFM- 
Table 1 Baseline characteristics before and after matching for the rVIII-SingleChain (phase I/III IPD) and rAHF-PFM-2004 prophylactic treatment populations $[16,28]$

\begin{tabular}{llllll}
\hline & \multicolumn{2}{l}{ Before matching } & & \multicolumn{2}{l}{ After matching } \\
\cline { 5 - 6 } & rVIII-SingleChain & rAHF-PFM-2004 & & rVIII-SingleChain & rAHF-PFM-2004 \\
\hline Number of subjects & $146^{\dagger}$ & 111 & $146^{\dagger}$ & 111 \\
Age > 18 years, \% & 84.2 & 44.1 & 44.1 & 44.1 \\
Race, \% white & 69.9 & 92.8 & 92.8 & 92.8 \\
Weight $(\mathrm{kg})$, mean & 74.0 & 65.8 & 65.8 & 65.8 \\
Height $(\mathrm{cm})$, mean & 174.4 & 169.3 & 169.3 & 169.3 \\
\hline
\end{tabular}

$I P D$ individual patient data, $r F V I I I$ recombinant factor VIII

$\dagger$ One patient was excluded in the comparison of the annualized rFVIII consumption because of missing data on this outcome. Baseline characteristics for these 145 patients can be found in Supplementary Material Table S2

* The effective sample size of rVIII-SingleChain after balancing with the rAHF-PFM-2004 population was 25.1

Table 2 Baseline characteristics before and after matching for the rVIII-SingleChain (phase I/III IPD) and rAHF-PFM-2012 prophylactic treatment population $[16,25]$

\begin{tabular}{llllll}
\hline & Before matching & & & After matching & \\
\cline { 2 - 3 } & rVIII-SingleChain & rAHF-PFM-2012 & & rVIII-SingleChain & rAHF-PFM-2012 \\
\hline Number of subjects & $146^{\dagger}$ & $32^{\ddagger}$ & $146^{\dagger}$ & $32^{\ddagger}$ \\
Age $\geq 16$ years, \% & 96.6 & 87.5 & 87.5 & 87.5 \\
Race, \% white & 69.9 & 93.8 & 93.8 & 93.8 \\
\hline
\end{tabular}

$I P D$ individual patient data, $r F V I I I$ recombinant factor VIII

$\dagger$ One patient was excluded in the comparison of the annualized rFVIII consumption because of missing data on this outcome. Baseline characteristics for these 145 patients can be found in Supplementary Material Table S3

* Standard prophylaxis arm

$\S$ The effective sample size of rVIII-SingleChain after balancing with the rAHF-PFM-2012 population was 79.0

2012 study; annualized rFVIII consumption as the total $\mathrm{rFVIIIFC}$ IU/kg received during the efficacy period divided by the number of days in the efficacy period times 365.25 in the A-LONG study) $[14,25,26]$. This was accommodated for by applying the same techniques to the IPD of the rVIII-SingleChain phase I/III trial. The resulting models and relative treatment effect measures for each outcome and each comparator trial can be found in Supplementary Material Table S5.

\section{rVIII-SingleChain Versus rAHF-PFM-2004}

Before matching, the mean ABR of rVIII-SingleChain was lower (3.43 vs 6.30) and the percentage of patients with zero bleeds higher (41.1\% vs $29.9 \%$ ) than those observed for rAHFPFM-2004. After matching, although less pronounced, the mean ABR of rVIII-SingleChain was still lower (4.64 vs 6.30) and the percentage of patients with zero bleeds still higher $(36.6 \%$ vs $29.9 \%$ ) than those of rAHF-PFM-2004. 
Table 3 Baseline characteristics before and after matching for the rVIII-SingleChain (phase I/III IPD) and rFVIIIFc prophylactic treatment population $[14,16]$

\begin{tabular}{|c|c|c|c|c|}
\hline & \multicolumn{2}{|l|}{ Before matching } & \multicolumn{2}{|l|}{ After matching } \\
\hline & rVIII-SingleChain & $\overline{\text { rFVIIIFc }}$ & rVIII-SingleChain $^{\#}$ & $\overline{\text { rFVIIIFc }}$ \\
\hline Number of subjects & $146^{\dagger}$ & $118^{\ddagger}$ & $146^{\dagger}$ & $118^{*}$ \\
\hline Age (years), mean & 29.7 & $29.0^{\S}$ & 29.0 & $29.0^{\S}$ \\
\hline Race, \% white & 69.9 & 66.9 & 66.9 & 66.9 \\
\hline Region, \% European & 47.3 & 28.8 & 28.8 & 28.8 \\
\hline Weight $(\mathrm{kg})$, mean & 74.0 & $74.0^{\circ}$ & 74.0 & $74.0^{\circ}$ \\
\hline
\end{tabular}

IPD individual patient data, $r F V I I I$ recombinant factor VIII

$\dagger$ One patient was excluded in the comparison of the annualized rFVIII consumption because of missing data on this outcome. Baseline characteristics for these 145 patients can be found in Supplementary Material Table S4

Individualized prophylaxis arm

$\$$ Mean age was estimated by the assumption that age follows a normal distribution

- Mean weight was estimated by the assumption that weight follows a lognormal distribution (reported median $71.65 \mathrm{~kg}$ )

\# The effective sample size of rVIII-SingleChain after balancing with the rFVIIIFc population was 123.6

Statistical significance was not reached in any of the comparisons (Fig. 1).

No data was available for rAHF-PFM-2004 that would allow for the comparison of annualized rFVIII consumption.

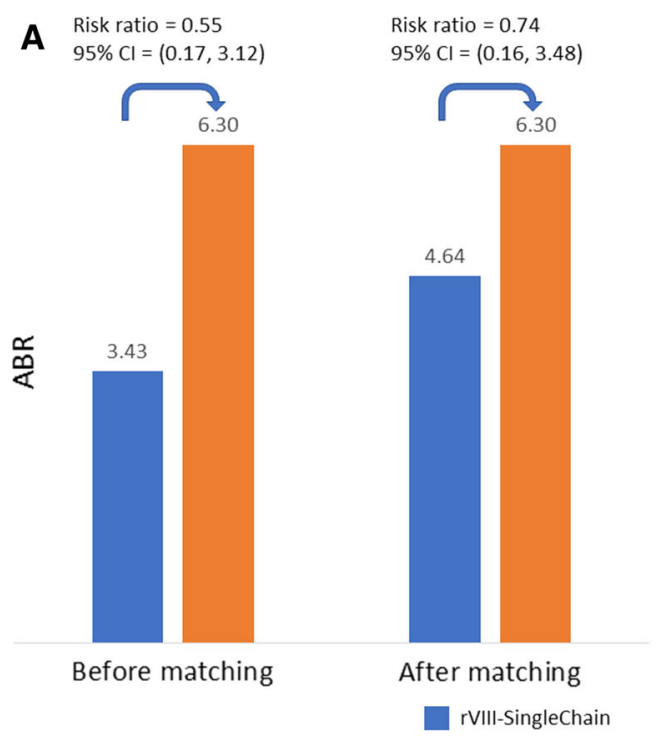

Fig. 1 Indirect comparison of rVIII-SingleChain versus rAHF-PFM-2004 for a mean ABR and $\mathbf{b}$ percentage of patients with zero bleeds, before and after matching. $P$ values for comparisons after matching were 0.70 for $A B R$

\section{rVIII-SingleChain Versus rAHF-PFM-2012}

The mean square root transformed ABR of rVIIISingleChain and rAHF-PFM-2012 were similar before and after matching (before, 1.67 vs 1.60 ;

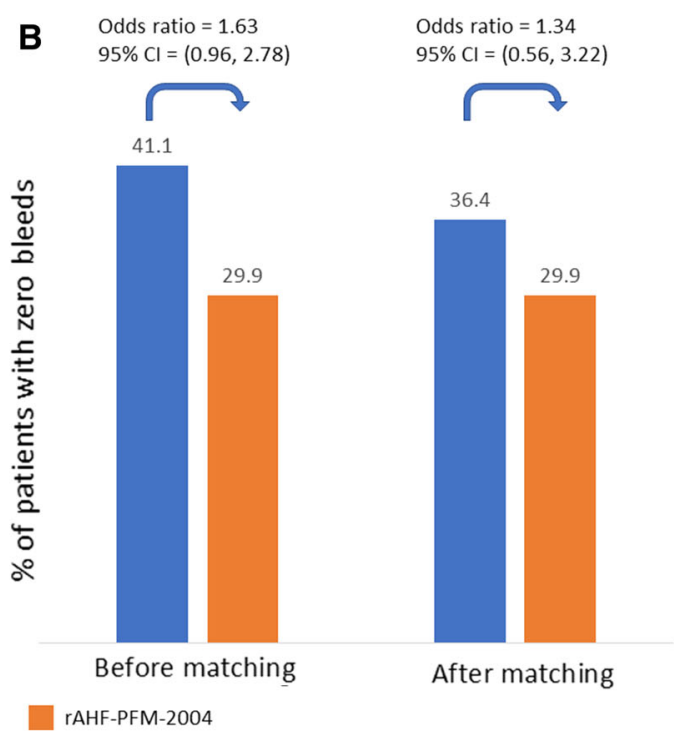

and 0.51 for percentage of patients with zero bleeds. $A B R$ annualized bleeding rate, $C I$ confidence interval 

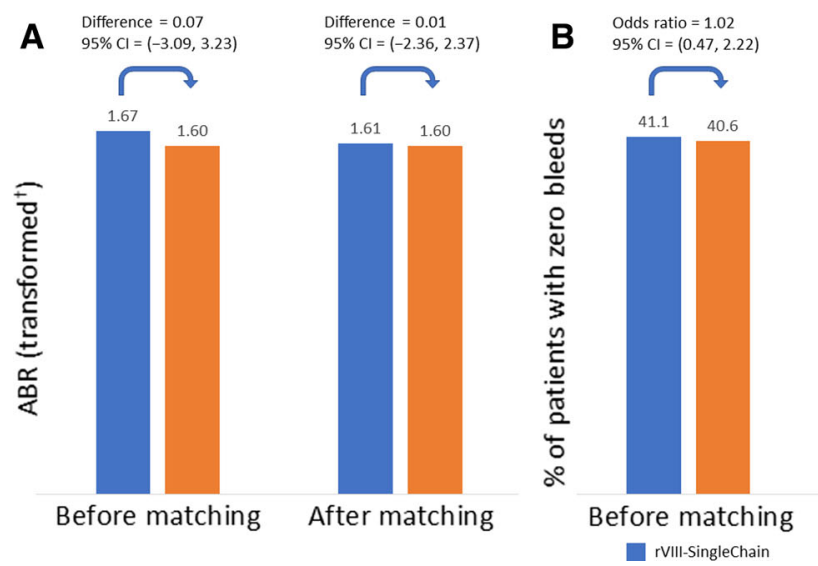

Fig. 2 Indirect comparison of rVIII-SingleChain versus rAHF-PFM-2012 for a mean square root transformed $\mathrm{ABR}^{\dagger}, \mathbf{b}$ percentage of patients with zero bleeds and $\mathbf{c}$ median annualized rFVIII consumption, before and after matching. ${ }^{\dagger}$ Transformed $\mathrm{ABR}=\sqrt{\mathrm{ABR}+0.5} . P$ values for comparisons after matching were 0.99 for $A B R, 0.91$

after, 1.61 vs 1.60$)$, as was the percentage of patients with zero bleeds (before, $41.1 \%$ vs $40.6 \%$; after, $41.8 \%$ vs $40.6 \%$ ) and median annualized rFVIII consumption (before, 4298 vs $5768 \mathrm{IU} / \mathrm{kg} /$ year; after, 4261 vs $5768 \mathrm{IU} / \mathrm{kg} /$ year).

For the square root transformed ABR and the percentage of patients with zero bleeds, none of the comparisons reached statistical significance (Fig. 2). The difference in median annualized rFVIII consumption, however, reached statistical significance $(P<0.0001)$ both before and after matching (Fig. 2). After matching, a median difference of $1508 \mathrm{IU} / \mathrm{kg} /$ year $(95 \%$ CI: $1004,2012 \mathrm{IU} / \mathrm{kg} /$ year) was estimated in favour of rVIII-SingleChain.

\section{rVIII-SingleChain Versus rFVIIIFc}

Before matching, rVIII-SingleChain was associated with a slightly higher estimated mean ABR (3.30 vs 2.91) and a slightly lower percentage of patients with zero bleeds (41.1\% vs $45.3 \%$ ) and mean annualized rFVIII consumption (4549 vs $4632 \mathrm{IU} / \mathrm{kg} /$ year) than rFVIIIFc. Comparisons after matching were similar to those before matching: 3.44 vs 2.91 (estimated mean ABR), $39.4 \%$ vs $45.3 \%$ (percentage of patients with zero bleeds), and 4444 vs $4632 \mathrm{IU} / \mathrm{kg} /$ year
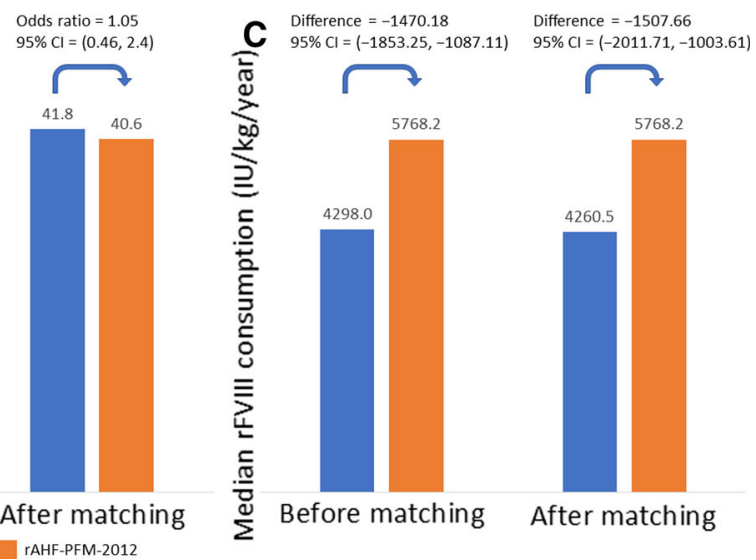

for percentage of patients with zero bleeds and $<0.0001$ for annualized rFVIII consumption. $A B R$ annualized bleeding rate, $C I$ confidence interval, $r F V I I I$ recombinant factor VIII

(annualized rFVIII consumption). None of the comparisons reached statistical significance (Fig. 3).

\section{DISCUSSION}

Interest in rFVIII replacement therapies for the prophylaxis of bleeding episodes in haemophilia A has been growing over the last decade, owing to the fact that they offer technological solutions to improve PK properties with the aim of prolonging intravenous injection intervals [11]. In the literature, a lot of focus has been placed on so-called extended half-life rFVIII products, engineered to have significantly extended plasma half-lives as compared to well-established standard-acting rFVIII products with plasma half-lives typically in the order of $12 \mathrm{~h}$ [29]. Although the half-life of a drug is acknowledged to be an important property, it has long been recognized that comparing different drugs by single PK parameters (like their half-lives) can be misleading, as they may not be representative of the overall PK profile [20]. A further issue with considering half-life as the most important PK parameter for rFVIII products in haemophilia $\mathrm{A}$ is that clearance of rFVIII is regulated to a large extent by 

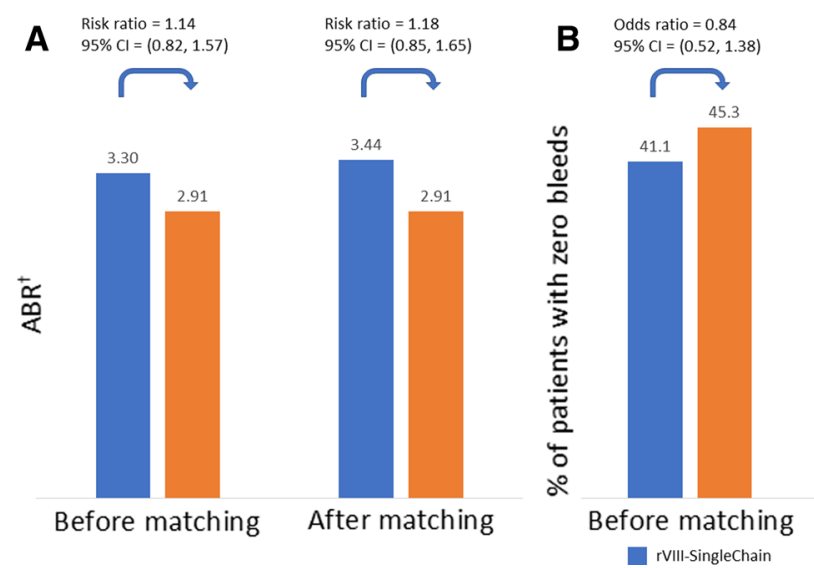

Fig. 3 Indirect comparison of rVIII-SingleChain versus rFVIIIFc for a estimated mean $\mathrm{ABR}^{\dagger}, \mathbf{b}$ percentage of patients with zero bleeds and $\mathbf{c}$ mean annualized rFVIII consumption, before and after matching. ${ }^{\dagger}$ Estimated by negative binomial regression model. (Total rFVIIIFc $\mathrm{IU} / \mathrm{kg}$ received during the efficacy period/number of days

interaction with VWF. As such, even with extended half-life rFVIII products, the maximum half-life achievable is currently limited by the half-life of VWF [30].

Since rVIII-SingleChain (lonoctocog alfa, AFSTYLA $^{\circledR}$, CSL Behring) only demonstrates a modest increase in half-life as compared to standard-acting rFVIII products [15], recent reviews of third-generation rFVIII products have suggested it does not qualify as an extended half-life rFVIII product [29, 31]. Owing to its high affinity for VWF, however, it achieves a much lower clearance rate, leading to a substantial increase in AUC, a greater drug exposure and the potential for longer dosing intervals than standard-acting rFVIII products, ultimately qualifying as a long-acting rFVIII product [11].

Some of the patients receiving prophylaxis with rVIII-SingleChain in the AFFINITY clinical program, with dosing regimens determined by the clinician on the basis of patient baseline characteristics and clinical phenotype, did receive a dosing regimen with injections at intervals of $2-3$ days [16, 17]. Nevertheless, a high proportion of patients were able to extend their dosing interval and reduce rFVIII
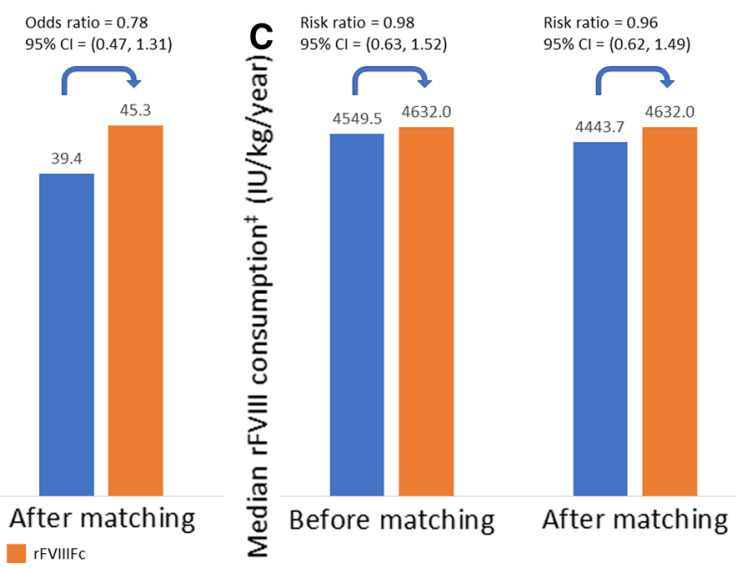

in efficacy period) $\times 365.25 . P$ values for comparisons after matching were 0.32 for ABR, 0.35 for percentage of patients with zero bleeds and 0.85 for annualized rFVIII consumption. $A B R$ annualized bleeding rate, $C I$ confidence interval, $r F V I I I$ recombinant factor VIII

consumption compared with pre-study standard-acting FVIII regimens [16, 17], further testifying to the long-acting nature of rVIIISingleChain.

Our study demonstrates that prophylactic treatment with rVIII-SingleChain has comparable efficacy to rAHF-PFM (octocog alfa, Advate ${ }^{\circledR}$, Shire), but with significantly lower consumption. Whereas comparable efficacy and consumption were established when compared to rFVIIIFc (efmoroctocog alfa, Elocta ${ }^{\circledR}$, Sobi).

All estimates on ABR, percentages of patients with zero bleeds and rFVIII consumption were obtained through MAIC, which, in the absence of head-to-head RCT (the gold standard for comparative evidence generation), still provides a clear advantage over naïve comparisons $[23,24]$. For example, a recent naïve comparison by Mannucci and colleagues stated that it is impossible to draw conclusions on the superiority of one product versus another using this approach; additionally, different prophylaxis regimens or patient selection can also make comparisons challenging [32]. By using individual patient-level data from the adolescent/ adult patient population of the rVIII-SingleChain phase I/III trial, MAIC corrected for 
potential confounding due to differences in baseline characteristics in common between study populations, leading to more balanced patient populations, typically observed as a result of randomization in RCT.

Limitations of our study are in line with those reported in comparable studies to inform real-world clinical decision-making on prophylactic treatment with other rFVIII in severe haemophilia A, using MAIC as the preferred population-adjusted method to generate comparative evidence [33-36]. While through the MAIC approach potential confounding due to imbalance in published and available baseline characteristics can be corrected for, residual confounding might still exist in baseline characteristics unavailable either for the rVIII-SingleChain prophylaxis arm and/or the prophylaxis arms of the comparator trials (e.g. type of previous FVIII replacement therapy). Furthermore, certain differences in trial designs leading to a lack of overlap in patient populations, especially concerning the older rAHF-PFM trials, could not be corrected for either: rAHFPFM trials allowed patients with moderately severe haemophilia A (rAHF-PFM-2004, 3.6\%; rAHF-PFM-2012, 6.2\%) and slightly lower minimum age; the rAHF-PFM-2012 trial only allowed patients with previous on-demand treatment $[25,28]$. Finally, the definitions of potential follow-up periods, important for the interpretation of non-annualized outcome measures, were inconsistent across the different clinical trials: a mean efficacy evaluation period of 244 days for rVIII-SingleChain; a mean total exposure of 117 days for rAHF-PFM-2004; a mean treatment period of 362 days for rAHFPFM-2012; and a median duration of treatment of 225 days for rFVIIIFc [14, 25, 28].

It is unclear how these and other factors bias results in favour of rVIII-SingleChain or its comparators. In the absence of head-to-head RCT, indirect comparisons such as ours are required. This highlights the need to better align clinical trials for haemophilia with respect to existing and/or new guidelines and the type of baseline characteristics and outcome measures collected.

\section{CONCLUSION}

The indirect treatment comparisons presented in this study contribute to the better understanding of the efficacy and factor consumption of rVIII-SingleChain as routine prophylaxis in previously treated adolescents/adults with severe haemophilia A, as compared to two other established and/or emerging rFVIII products. Results suggest that with an annualized rFVIII consumption comparable to rFVIIIFc, but significantly lower than rAHF-PFM, routine prophylaxis with rVIII-SingleChain is able to maintain a similar annualized bleeding rate and percentage of patients with zero bleeds, attesting to the long-acting nature of rVIIISingleChain.

\section{ACKNOWLEDGEMENTS}

Funding. This study was funded by CSL Behring, Hattersheim, Germany. The Rapid Service and Open Access Fees were also funded by CSL Behring.

Medical Writing and Editorial Assistance. Medical writing and editorial support (in the form of writing assistance, grammatical editing, submission support) was provided by Sarah Angus, PhD, Meridian HealthComms Ltd, Plumley, UK, and was funded by CSL Behring.

Authorship. All named authors meet the International Committee of Medical Journal Editors (ICJME) criteria for authorship for this article, take responsibility for the integrity of the work as a whole, and have given their approval for this version to be published.

Author Contributions. SB: critical revision of the manuscript; RN: critical revision of the manuscript; JLP: critical revision of the manuscript; KK: critical revision of the manuscript; GG: critical revision of the manuscript; VA: data acquisition, analysis and data interpretation, critical revision of the manuscript; BV: data acquisition, analysis and data interpretation, drafting and critical revision of the manuscript; 
SS: study conception and design, drafting and critical revision of the manuscript.

Prior Presentation. An abstract containing some of the data on which this manuscript is based was presented at the European Association for Haemophilia and Allied Disorders (EAHAD) 2020 annual congress held in The Hague, Netherlands, 4-7 February.

Disclosures. Santiago Bonanad has acted as a paid speaker and consultant, and received grants for attending congresses; Ramiro Núñez reports personal fees or consulting services for Novo Nordisk, Takeda, Pfizer, Bayer, Sobi, Roche and CSL Behring; Jose Luis Poveda has acted as a paid consultant to Chiesi; Karin Kurnik received grants, travel support and/or lecture fees from CSL Behring, Shire/Takeda and Sobi, and honoraria for advisory boards from CSL Behring and Shire/Takeda; Georg Goldmann has undertaken speaker duties for Bayer, Shire, Pfizer, CSL Behring, Novo Nordisk, Sobi, Biotest, Nordic and Octapharma, has acted as a consultant for Bayer, Shire, Pfizer, CSL Behring, Novo Nordisk, Sobi, BPL and Octapharma, and has received research funding from Bayer, Shire, Pfizer, CSL Behring, Novo Nordisk and Sobi; Valeska Andreozzi and Björn Vandewalle are employed by Exigo Consultores: CSL Behring contracted with Exigo Consultores for the development of the research project and Exigo Consultores provided support in the form of salaries for authors but did not have any additional role in the study design, data collection and analysis, or preparation of the manuscript; Sandra Santos is an employee of CSL Behring.

Compliance with Ethics Guidelines. The phase I/III clinical trial of rVIII-SingleChain was conducted in accordance with the International Conference on Harmonization Guidelines for Good Clinical Practice and the ethical principles outlined in the Declaration of Helsinki 2008. Ethics approval, individual informed consent, and approval by the relevant national authorities were obtained prior to enrolment. Informed consent was not required for this analysis given the deidentified nature of the AFFINITY individualized patient-level data and the use of anonymized, previously published data for rAHF-PFM and rFVIIIFc studies.

Data Availability. The datasets generated during and/or analyzed during the current study are already publicly available in the published manuscripts. Individual patient-level data (IPD) used in this analysis were obtained from the trial sponsor. For information on the process and requirements for submitting a voluntary data sharing request for IPD, please contact the trial sponsor at clinicaltrials@cslbehring.com.

Open Access. This article is licensed under a Creative Commons Attribution-NonCommercial 4.0 International License, which permits any non-commercial use, sharing, adaptation, distribution and reproduction in any medium or format, as long as you give appropriate credit to the original author(s) and the source, provide a link to the Creative Commons licence, and indicate if changes were made. The images or other third party material in this article are included in the article's Creative Commons licence, unless indicated otherwise in a credit line to the material. If material is not included in the article's Creative Commons licence and your intended use is not permitted by statutory regulation or exceeds the permitted use, you will need to obtain permission directly from the copyright holder. To view a copy of this licence, visit http://creativecommons.org/licenses/by$\mathrm{nc} / 4.0 /$.

\section{REFERENCES}

1. Rodriguez-Merchan EC, Valentino LA. Orthopedic disorders of the knee in hemophilia: a current concept review. World J Orthop. 2016;7(6):370-5. https://doi.org/10.5312/wjo.v7.i6.370:10.5312/wjo. v7.i6.370.

2. Auerswald G, Dolan G, Duffy A, et al. Pain and pain management in haemophilia. Blood Coagul Fibrinolysis. 2016;27(8):845-54. https://doi.org/10. 1097/MBC.0000000000000571:10.1097/MBC.0000 000000000571.

3. Srivastava A, Santagostino E, Dougall A, et al. WFH guidelines for the management of hemophilia, 3rd 
edition. Haemophilia. 2020;26(Suppl 6):1-158. https://doi.org/10.1111/hae.14046.

4. O'Mahony B, Noone D, Giangrande PL, Prihodova L. Haemophilia care in Europe-a survey of 35 countries. Haemophilia. 2013;19(4):e239-247. https://doi.org/10.1111/hae.12125.

5. Gringeri A. Factor VIII safety: plasma-derived versus recombinant products. Blood Transfus. 2011;9(4): 366-70. https://doi.org/10.2450/2011.0092-10.

6. Schiavoni M, Napolitano M, Giuffrida G, et al. Status of recombinant factor VIII concentrate treatment for hemophilia A in Italy: characteristics and clinical benefits. Front Med (Lausanne). 2019;6: 261. https://doi.org/10.3389/fmed.2019.00261.

7. European Medicines Agency. Advate (octocog alfa). Authorisation details. https://www.ema.europa.eu/ en/medicines/human/EPAR/advate\#authorisationdetails-section. Accessed May 2021.

8. Takeda Manufacturing Austria AG. Advate Summary of Product Characteristics 2020. https://www. ema.europa.eu/en/documents/product-information/ advate-epar-product-information_en.pdf. Accessed May 2021.

9. Tiede A. Half-life extended factor VIII for the treatment of hemophilia A. J Thromb Haemost. 2015;13(Suppl 1):S176-179. https://doi.org/10. $1111 /$ jth. 12929 .

10. Berntorp E. Joint outcomes in patients with haemophilia: the importance of adherence to preventive regimens. Haemophilia. 2009;15(6):1219-27. https://doi.org/10.1111/j.1365-2516.2009.02077.x.

11. Escobar M, Santagostino E, Mancuso ME, et al. Switching patients in the age of long-acting recombinant products? Expert Rev Hematol. 2019;12(sup1):1-13. https://doi.org/10.1080/

17474086.2018.1564032.

12. Swedish Orphan Biovitrum AB. Elocta Summary of Product Characteristics 2019. https://www.ema. europa.eu/en/documents/product-information/elo cta-epar-product-information_en.pdf. Accessed May 2021.

13. European Medicines Agency. Elocta (efmoroctocog alfa). Authorisation details. https://www.ema. europa.eu/en/medicines/human/EPAR/elocta\#auth orisation-details-section. Accessed May 2021.

14. Mahlangu J, Powell JS, Ragni MV, et al. Phase 3 study of recombinant factor VIII Fc fusion protein in severe hemophilia A. Blood. 2014;123(3):317-25. https://doi.org/10.1182/blood-2013-10-529974.
15. Klamroth R, Simpson M, von Depka-Prondzinski M, et al. Comparative pharmacokinetics of rVIII-SingleChain and octocog alfa (Advate(®) ) in patients with severe haemophilia A. Haemophilia. 2016;22(5):730-8. https://doi.org/10.1111/hae. 12985.

16. Mahlangu J, Kuliczkowski K, Karim FA, et al. Efficacy and safety of rVIII-SingleChain: results of a phase 1/3 multicenter clinical trial in severe hemophilia A. Blood. 2016;128(5):630-7. https:// doi.org/10.1182/blood-2016-01-687434.

17. Stasyshyn O, Djambas Khayat C, Iosava G, et al. Safety, efficacy and pharmacokinetics of rVIII-SingleChain in children with severe hemophilia A: results of a multicenter clinical trial. J Thromb Haemost. 2017;15(4):636-44.

18. ClinicalTrials.gov. An open-label safety and efficacy study of recombinant FVIII in patients with severe hemophilia A (NCT02172950). https:// clinicaltrials.gov/ct2/show/NCT02172950. Accessed May 2021.

19. Zollner S, Raquet E, Claar P, et al. Non-clinical pharmacokinetics and pharmacodynamics of rVIIISingleChain, a novel recombinant single-chain factor VIII. Thromb Res. 2014;134(1):125-31. https://doi.org/10.1016/j.thromres.2014.03.028.

20. Greenblatt DJ. Elimination half-life of drugs: value and limitations. Annu Rev Med. 1985;36:421-7. https://doi.org/10.1146/annurev.me.36.020185. 002225 .

21. Gringeri A, Ewenstein B, Reininger A. The burden of bleeding in haemophilia: is one bleed too many? Haemophilia. 2014;20(4):459-63. https://doi.org/ 10.1111/hae. 12375 .

22. CSL Behring. AFSTYLA Summary of Product Characteristics 2019. https://www.ema.europa.eu/en/ documents/product-information/afstyla-epar-prod uct-information_en.pdf. Accessed May 2021.

23. Ishak KJ, Proskorovsky I, Benedict A. Simulation and matching-based approaches for indirect comparison of treatments. Pharmacoeconomics. 2015;33(6):537-49. https://doi.org/10.1007/s40273015-0271-1.

24. Phillippo DM, Ades AE, Dias S, Palmer S, Abrams KR, Welton NJ. Methods for population-adjusted indirect comparisons in health technology appraisal. Med Decis Making. 2018;38(2):200-11. https:// doi.org/10.1177/0272989X17725740.

25. Valentino LA, Mamonov V, Hellmann A, et al. A randomized comparison of two prophylaxis regimens and a paired comparison of on-demand and prophylaxis treatments in hemophilia A 
management. J Thromb Haemost. 2012;10(3): 359-67. https://doi.org/10.1111/j.1538-7836.2011. 04611.x.

26. ClinicalTrials.gov. A-LONG: an open-label, multicenter evaluation of the safety, pharmacokinetics, and efficacy of recombinant factor VIII Fc fusion protein (rFVIIIFc) in the prevention and treatment of bleeding in previously treated subjects with severe hemophilia A. Study results. https:// clinicaltrials.gov/ct2/show/results/NCT01181128. Accessed May 2021.

27. R Core Team. R: a language and environment for statistical computing [computer program]. 2019. Vienna: R Foundation for Statistical Computing.

28. Tarantino MD, Collins PW, Hay CR, et al. Clinical evaluation of an advanced category antihaemophilic factor prepared using a plasma/albumin-free method: pharmacokinetics, efficacy, and safety in previously treated patients with haemophilia A. Haemophilia. 2004;10(5):428-37. https://doi.org/10.1111/j.1365-2516.2004.00932.x.

29. Lambert T, Benson G, Dolan G, et al. Practical aspects of extended half-life products for the treatment of haemophilia. Ther Adv Hematol. 2018;9(9):295-308. https://doi.org/10.1177/ 2040620718796429.

30. Pipe SW, Montgomery RR, Pratt KP, Lenting PJ, Lillicrap D. Life in the shadow of a dominant partner: the FVIII-VWF association and its clinical implications for hemophilia A. Blood. 2016;128(16):2007-16. https://doi.org/10.1182/ blood-2016-04-713289.

31. Mahlangu J, Young G, Hermans C, Blanchette V, Berntorp E, Santagostino E. Defining extended half- life rFVIII-a critical review of the evidence. Haemophilia. 2018;24(3):348-58.

32. Mannucci PM, Cortesi PA, Di Minno MND, Sano M, Mantovani LG, Di Minno G. Comparative analysis of the pivotal studies of extended half-life recombinant FVIII products for treatment of haemophilia A. Haemophilia. 2021;27(4):e422-33. https://doi. org/10.1111/hae.14313.

33. Pocoski J, Li N, Ayyagari R, et al. Matching-adjusted indirect comparisons of efficacy of BAY 81-8973 vs two recombinant factor VIII for the prophylactic treatment of severe hemophilia A. J Blood Med. 2016;7:129-37. https://doi.org/10.2147/JBM. S104074.

34. Ayyagari R, Deschaseaux C, Vashi P, Kessabi S, Gao W. Matching-adjusted indirect comparisons (MAICs) of efficacy and consumption of BAY 94-9027 versus three recombinant factor VIII (rFVIII) for prophylaxis of severe hemophilia A. Value Health. 2018;21:S246.

35. Batt K, Gao W, Ayyagari R, et al. Matching-adjusted indirect comparisons of annualized bleeding rate and utilization of BAY 94-9027 versus three recombinant factor VIII agents for prophylaxis in patients with severe hemophilia A. J Blood Med. 2019;10:147-59. https://doi.org/10.2147/JBM. S206806.

36. Hakimi Z, Santagostino E, Postma MJ, Nazir J. Recombinant FVIIIFc versus BAY 94-9027 for treatment of patients with haemophilia A: comparative efficacy using a matching adjusted indirect comparison. Adv Ther. 2021;38(2):1263-74. https://doi.org/10.1007/s12325-020-01599-1. 